\title{
Structure of thin cobalt films on Copper studied by EXAFS and analyzed with the FEFF code
}

\author{
P. Le Fèvre, D. Chandesris, H. Magnan* and O. Heckmann** \\ LURE, Université Paris Sud, 91405 Orsay, France \\ * CEA-DRECAM-SRSIM \\ ** Université de Cergy Pontoise, France
}

\begin{abstract}
A polarization dependent first shell analysis of the EXAFS spectra shows that cobalt grows on $\mathrm{Cu}(100)$ and $\mathrm{Cu}(111)$ in perfect epitaxy : the mean nearest neighbour distance between cobalt atoms parallel to the surface is $2.55 \AA$ (same value as in bulk copper) and the interlayer bond length is $2.50 \AA$. The contraction of the lattice parameter perpendicular to the surface is in agreement with the continuum elasticity theory.

The analysis of further neighbour shells is done with the FEFF code (multiple scattering) modified to take into account the structural anisotropy. Simulations are compared to experiments and show anumbiguously that cobalt has an fcc tetragonal structure on $\mathrm{Cu} \mathrm{100,} \mathrm{but} \mathrm{a} \mathrm{distorted} \mathrm{hep} \mathrm{structure}$ on $\mathrm{Cu}(111)$. The (111) surface of copper is hexagonal. An fcc structure means an ..ABCABC.. stacking, and an hcp structure an ..ABAB.. stacking. The main difference between these two structures is the absence of alignment of the first and fourth neighbours in the hep structure in grazing incidence. This effect is clear in the calculated and in the experimental Fourier Transforms of the EXAFS spectra.
\end{abstract}

\section{INTRODUCTION}

Thin metastable films of cobalt grown on copper are the subject of many studies for the last few years because of their original magnetic properties as free films or in multilayers. To understand the magnetic properties of this kind of metastable films, it is obvious that it is necessary to know precisely their cristallographic structure. In this paper, we present a surface EXAFS study of thin cobalt films on $\mathrm{Cu}(001)$ and $\mathrm{Cu}(111)$. By performing a Fourier transform (FT) of the EXAFS spectra, we obtain a series of peaks due to the different shells of neighbours of the absorbing atom. The first nearest neighbour peak is isolated and backtransformed. This contribution of the first shell to the EXAFS signal is then fitted using the classical formula of EXAFS calculated in the single scattering formalism [1]. It gives to a high precision first nearest neighbour distances. The linear polarization of the synchrotron radiation is used to isolate the contribution to the EXAFS signal of the different kinds of bonds: in-plane or out-of-plane bonds. It allows to measure the structural anisotropy of the films. Moreover, the shape of the FT of the EXAFS spectra is also strongly polarization dependent. The simulation of these higher shells signals are no more possible in a single scattering formalism, and requires a multiple scattering calculation. We have used the FEFF code developed by J.J. Rehr and al. to confirm and complete the first shell analysis in the $\mathrm{Co} / \mathrm{Cu}(001)$ and $\mathrm{Co} / \mathrm{Cu}(111)$ cases. The materials in this paper is organised as follows. In section 2 , the experimental procedure is quickly described.We present our results for cobalt films deposited on $\mathrm{Cu}(001)$ in section 3 , and in section 4 for cobalt films on $\mathrm{Cu}(111)$.

\section{EXPERIMENTAL PROCEDURE}

The EXAFS experiments are performed at the Laboratoire pour l'Utilisation du Rayonnement Electromagnétique (LURE) on the surface EXAFS set-up using a Si (311) double crystal monochromator 
installed on the wiggler beam line of DCI storage ring. The two copper surfaces are prepared by long argon ionic bombardment of the crystals maintained at about $500^{\circ} \mathrm{C}$. The crystallographic quality of the surfaces is checked by LEED and their cleanliness by Auger spectroscopy. Cobalt is evaporated from a high purity wire heated by electron bombardment in a vacuum better than $5 \times 10^{-10}$ mbar (base pressure is $2 \times 10^{-10}$ mbar). It is deposited at a rate of about 1 monolayer per minute at room temperature on a clean copper surface. The local atomic structure of the films is then studied in situ. The EXAFS measurements are performed on samples maintained at $77 \mathrm{~K}$ in order to reduce the thermal disorder and improve the precision of the crystallographic characterization of the cobalt films.

The variations of the X-ray absorption coefficient of the samples are measured above the K edge of cobalt $(7709 \mathrm{eV})$ in the total yield mode. EXAFS spectra are recorded both in normal incidence (electric field of the X-rays parallel to the surface) and in grazing incidence (electric field is about $20^{\circ}$ from the surface normal).

\section{3. $\mathrm{Co} / \mathrm{Cu}(001)$}

\subsection{Introduction}

Thin metastable films of cobalt deposited on $\mathrm{Cu}(001)$ have already been investigated by several techniques. Angle resolved X ray photoemission scattering [2] and MEED oscillations combined with in situ STM experiments [3] show that cobalt grows to a well ordered fcc(001) film via a layer-by-layer growth mode, except for the first two layers. The films are always ferromagnetic, independent of the film thickness, and the remanent magnetization is always parallel to the interface [4], with a strong in-plane anisotropy : the [110] direction is the easy axis $[5,6,7]$. The Curie temperature of films evaporated at $450 \mathrm{~K}$ is strongly increasing with the film thickness from $130 \mathrm{~K}$ for $1.5 \mathrm{ML}$ to $500 \mathrm{~K}$ for $2.5 \mathrm{ML}$ [5].

The interpretation of the photoemission spectra [8] and of the magnetic properties [9] is often made assuming a tetragonal distortion of the structure. But the supposed tetragonalization has not been precisely measured for the whole Co film and for different thicknesses. One can quote a quantitative LEED analysis which indicates that cobalt epitaxially grows on $\mathrm{Cu}(001)$ with an fcc tetragonal structure [9]. But the sensitivity of LEED decreases very quickly with the depth: it characterizes only the first two or three surface layers, and the information in the direction perpendicular to the surface is indirect (it requiers a full multiple scattering calculation). We have studied thin cobalt films from 2 to 15 monolayers (ML).

\subsection{First shell analysis}

A first remark is that the shape of the FT of the EXAFS spectra is different in normal and in grazing incidence. In our case, if we assume a perfect fcc epitaxy, every cobalt atom has 4 nearest neighbours (nn) in its plane, 4 above (missing for the top layer) and 4 below. When the polarization of the light is perpendicular to the surface (grazing incidence) only the interlayer bonds contribute to the EXAFS signal but in normal incidence (polarization parallel to the surface) both interlayer bonds and intralayer bonds are contributing with the same weight. This polarization dependence of the shape of the FT is therefore a first proof of the anisotropy of the films. The first shell analysis confirms this observation.

For each sample, we begin by a fitting of the grazing incidence spectrum assuming a single shell of neighbours, since the interlayer bonds are the only one which give a significant contribution. Then, the fitting procedure is applied to the normal incidence spectra where the intralayer bonds and the interlayer ones contribute with about the same weight.We fix the interlayer bond length and Debye-Waller factors to their values measured in grazing incidence, and then, in the fitting procedure, the free parameters are just the nn intralayer bond length and its associated Debye-Waller factor. For all the samples, the in plane $\mathrm{nn}$ distance is $2.55 \AA( \pm 0.01 \AA)$ which is exactly the nn distance in bulk copper, and the out-of plane $n n$ distance is $2.50 \AA( \pm 0.01 \AA)$. So the tetragonal deformation of the films appears to be an expansion of the fcc crystal parallel to the interface to adapt exactly the in-plane nn distance of the film to substrate's one.

\subsection{Simulations of the spectra using the FEFF code}

From this first shell analysis, one can assume that the cobalt films have an fcc tetragonalized structure. In the FT of the EXAFS spectra, we see well defined peaks up to a distance of $6 \AA$; they are due to single scattering on neighbours up to the sixth shell and to multiple scattering with paths of the same lengths. The position and intensity of these FT peaks are very sensitive to the structure : we shall confirm (or not) the tetragonal fcc structure of the films, if we are able (or not) to reproduce them on EXAFS data calculated using a multiple scattering formalism. In particular, the experimental Fourier transforms are strongly anisotropic: can this anisotropy be reproduced only considering the tetragonalization that we deduced from the first shell analysis?

We have used the FEFF code, developed by J.J. Rehr et al. (Version 5.04) [10]. In this version, is not possible to take into account the linear polarization of the light : the program assumes that the system is isotropic. Since our system is strongly anisotropic and the synchrotron radiation is linearly polarized in our 
$\mathrm{Co} / \mathrm{Cu}(100)$ $\mathrm{Co} / \mathrm{Cu}(111)$

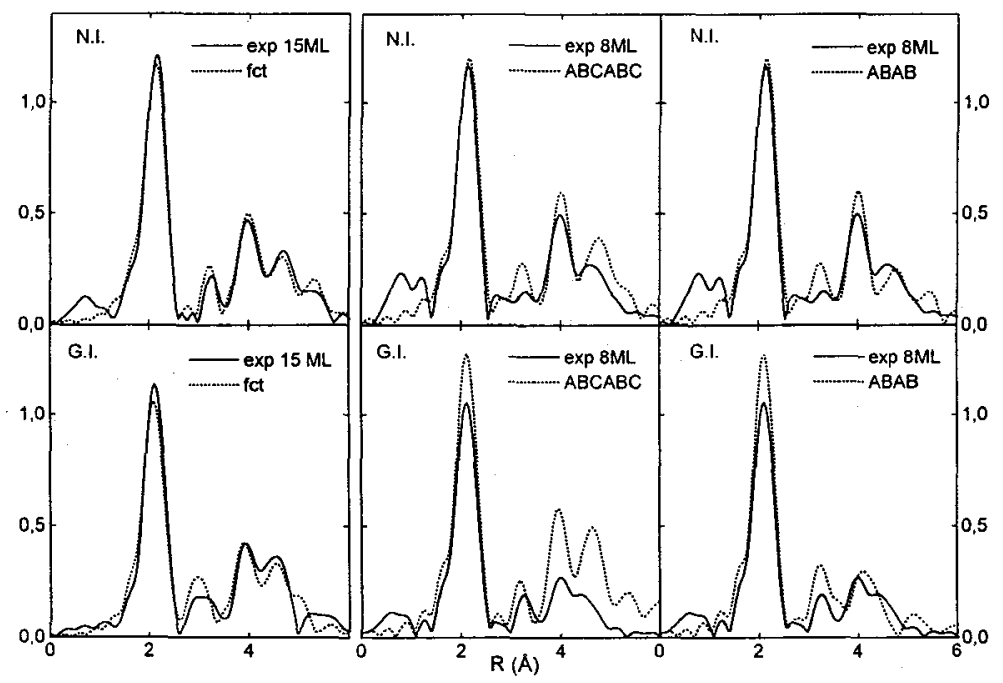

Figure 1 : Fourier Transforms of the EXAFS spectra recorded in Normal Incidence (N.I.) and in Grazing Incidence (G.I.) (solid line) compared to multiple scattering simulations (dashed line) using the FEFF code.

experiment, we have introduced the polarization dependence in an approximated way. We have assumed that, for each geometry (grazing incidence and normal incidence) the relative contribution of each path (single scattering and multiple scattering ones) could be approximated by its relative weight calculated in the plane wave approximation. The validity of this method was succesfully tested on a well known non isotrope sample: a monocrystal of $\mathrm{Co}(0001)$. We have then calculated, with the same method, the polarization dependent EXAFS spectra of cobalt clusters with a fcc tetragonal structure. It appears that the shape of FT of the simulations is clearly changing when varying the tetragonalization, thus proving the sensitivity of the method to the amplitude of distortion.

We then compare the FEFF simulations with the experimental FT for $\mathrm{Co} / \mathrm{Cu}(001)$ films. Since the simulations are made for bulk crystals without surfaces, we consider the experimental results on the thicker film: $15 \mathrm{ML}$. The best agreement is obtained for a structure with a nn distance of $2.56 \AA$ in plane and of $2.50 \AA$ out of plane, very close to those deduced from the first shell analysis. The FEFF calculations reproduce very well most of the positions, intensities and polarization dependence of the different peaks of the FT. The cobalt films are in perfect epitaxy on $\mathrm{Cu}(001)$. First shell analysis, and multiple scattering simulations demonstrate the fct structure of the films.

\section{4. $\mathrm{Co} / \mathrm{Cu}(111)$}

\subsection{Introduction}

The growth of cobalt films on $\mathrm{Cu}(111)$ seems to be very different from those on $\mathrm{Cu}(001)$. A recent STM study reports observations of granular films made of domains twinned by $60^{\circ}$ [11]. As a matter of fact, it is difficult to predict what structure the cobalt films are going to choose.

Copper is fcc, and the [111] axis is the stacking direction of the compact planes with a (...ABCABC...) period. Bulk cobalt is hcp, which is also a compact structure. A $(0001)$ oriented crystal will have the same surface lattice, and will only differ in the stacking period which will be (...ABAB...). The two structures are therefore very close. Will the cobalt continue the copper lattice and adopt an fcc structure, or will it grow with its hep bulk stable structure?

When cobalt is deposited, the threefold symetry of the LEED pattern of the clean $\mathrm{Cu}(111)$ surface changes to a sixfold symetry pattern [12], but this could result from a non-flat (0001) hcp surface, or from a twinned fcc(111) film [13]. Two photodiffraction studies conclude to fcc stackings in the early stages of growth, and to a mainly hep structure for thicker films [13,14]. On the other hand, in Co-Cu superlattices, F.J. Lamelas et al. observed by X-rays diffraction metastable fec cobalt films with $35 \%$ of hcp domains [15]. 
We have studied films from 2 to $8 \mathrm{ML}$. We will first precisely determine the local order in the films by a first nearest neighbour shell analysis. We will then conclude on the structure of the films with polarization dependent simulations of the EXAFS spectra.

\subsection{First shell analysis}

Once again, the shapes of the FT of the EXAFS spectra are different in normal and in grazing incidence. This means that the cobalt films still have a non isotrope structure. Independently from the structure of the films, the first nearest neighbour shell has the same configuration. Every cobalt atom has six $\mathrm{nn}$ in its plane, three above (missing for the top layer), and three below. In grazing incidence, only the interlayer bonds are contributing to the EXAFS signal, but in normal incidence, the two types of bonds are contributing, the in-plane bonds having a three times larger weight.

The fitting procedure is the same than for $\mathrm{Co} / \mathrm{Cu}(001)$. A fit of the grazing incidence spectra allows to measure the length of the out-of-plane bonds; the result is then used to determine the in-plane nn distance by fitting the normal incidence spectra. For all the samples, we find a in-plane nn distance of $2.54 \AA$, and of $2.51 \AA$ for neighbours out of the surface plane. The first shell analysis does not allow to conclude on the cristallographic structure of the films. We have once again simulated the EXAFS spectra with a multiple scattering program.

\subsection{Simulations of the spectra using the FEFF code}

Are the cobalt films fcc or hcp? In the fcc structure, each first neighbour at a distance $R$ of the central atom is aligned with a fourth neighbour at a distance $2 \mathrm{R}$ of the central atom. This alignement enhances the intensity of the fourth shell in the FT, both in normal and in grazing incidence (focussing effect). On the other hand, in the hcp structure, this alignement exists in the (0001) basal planes, but is lost out of these planes. this absence of focussing in grazing incidence gives a strong polarization dependence of the fourth peak in the FT. The calculation was done with FEFF, version 6.0, which takes the polarization of the light into account in a curved wave formalism, with clusters constructed using the cristallographic paramaters determined from the first shell analysis. In normal incidence, the two FT of the simulations look alike and fit the experimental data in a quite equivalent way. But, as expected, the FT of the grazing incidence simulations have very different shapes. The lost of atoms alignement in the ...ABAB... stackings makes the higher shell peaks dramaticaly smaller. Unambigously, the agreement with experiment is much better for the ...ABAB ... simulation than for the ... ABCABC... one.

From this study, we can conclude that the cobalt films are in epitaxy on $\mathrm{Cu}(111)$. Their structure is mainly hep.

[1] D. E. Sayers, E. A. Stern and F. W. Lytle, Phys. Rev. Lett. 27 (1971) 1204.

[2] Hong Li and B. P. Tonner, Surf. Sci. 237 (1990) 141.

[3] C. M. Schneider, A.K. Schmid, H.P. Oepen and J. Kirschner, "Structure and Magnetism in Low Dimensional Systems", eds R.F.C. Farrow, M. Donath, B. Dieny, A. Fert, B. Hermsmeier (Plenum Press, New York 1993); A. K. Schmidt, J. Kirschner, Ultramicroscopy 42-44, (1992) 483.

[4] D. Pescia, G. Zampieri, M. Stampanoni, G. L. Bona, R. F. Willis, F. Meier, Phys. Rev. Lett. 58 (1987) 933.

[5] C. M. Schneider, P. Bressler, P. Schuster, J. Kirschner, J. J. de Miguel, and R. Miranda, Phys. Rev. Lett. 64 (1990) 1059.

[6] B. Heinrich, J.F. Cochran, M. Kowalewski, J. Kirschner, Z. Celinski, A.S. Arrott, and K. Myrtle, Phys. Rev. B 44 (1991) 9348; M. Kowalewski, C.M. Schneider and B. Heinrich, Phys. Rev. B 47 (1993) 8748.

[7] P. Krams, F. Lauks, R. L. Stamps, B. Hillebrands and G. Güntherodt, Phys. Rev. Lett. 69 (1992) 3674; H. P. Oepen, M. Benning, H. Xbach, C. M. Schneider, and J. Kirschner, Journal of Magn. Magn. Mat. 86 (1990) L 137.

[8] C. M. Schneider, P. Schuster, M. Hammond, H. Ebert, J. Noffke, J. Kirschner, J. Phys. Condensed Matter 3 (1991) 4349.

[9] A. Clarke, G. Jennings, R. F. Willis, P. J. Rous, and J. B. Pendry, Surf. Sci. 187 (1987) 327.

[10] J. J. Rehr, J. Mustre de Leon, S. I. Zabinsky, and R. C. Albers, J.Am. Chem. Soc. 113 (1991)

5135; J. Mustre de Leon, J. J. Rehr, S. I. Zabinsky, R. C. Albers, Phys. Rev. B 44 (1991) 4146; J. J.

Rehr, R. C. Alberts, S. I. Zabinsky Phys. Rev. Lett. 69 (1992) 3397.

[11] J. de la Figuera, J. E. Prieto, C. Ocal, and R. Miranda, Phys. Rev. B 47 (1993) 13043.

[12] L. Gonzalez, R. Miranda, M. Salmeron, J. A. Vergés, and F. Indurain, Phys. Rev. B 24 (1981) 3245 .

[13] B. P. Tonner, Z.-L. Han, and J. Zhang, Phys. Rev. B 47 (1993) 9723.

[14] Th. Fauster, G. Rangelov, J. Stober, and B. Eisenhut, Phys. Rev. B 48 (1993) 11361.

[15] F. J. Lamelas, C. H. Lee, H. He, W. Vavra, and Roy Clarke, Phys. Rev. B 40 (1989) 5837. 